\section{Symptomatisches Vorhofflimmern: Kryoballon als Alternative zur Radiofrequenzablation}

\author{
Bei Patienten mit refraktärem, symptomatischen Vorhofflimmern hat die \\ Kryoballon-Therapie Vorteile gegenüber der Radiofrequenzablation hinsicht- \\ lich erneuter Ablationsmaßnahmen, elektrischer Kardioversionen und der \\ Notwendigkeit erneuter Krankenhauseinweisungen.
}

D ie Studie FIRE AND ICE zeigte an über 750 Patienten mit therapierefraktärem, symptomatischem Vorhofflimmern, dass die Kryoballon-Therapie gegenüber der Radiofrequenzablation (RFC) hinsichtlich des primären Endpunkts (erstes Vorhofflimmerrezidiv, Vorhofflattern/atriale Tachykardie, Verwendung antiarrhythmischer Substanzen, erneute Ablation außerhalb eines 90-Tages-Fensters) vergleichbare Ergebnisse liefert.

In der aktuell vorgestellten Analyse der sekundären Endpunkte wurden für den klinischen Alltag relevante Ereignis- se miteinander verglichen (erneute $\mathrm{Ab}$ lationsmaßnahme, elektrische Kardioversionen und Notwendigkeit erneuter Krankenhauseinweisungen, Lebensqualität).

Im Ergebnis erfolgten in der mit Kryoballon behandelten Patientengruppe signifikant weniger Re-Ablationen. Zudem kam es zu weniger elektrischen Kardioversionen ( 3,2 vs. 6,4\%; $\mathrm{p}=0,04)$ und einer geringeren Rehospitalisierungsrate ( 32,6 vs. $41,5 \% ; p=0,01)$. Es gab keinen statistischen Unterschied hinsichtlich der Lebensqualität zwischen beiden Behandlungsgruppen.
Kuck KH et al. Cryoballoon or radiofrequency ablation for symptomatic paroxysmal atrial fibrillation: reintervention, rehospitalization, and quality-of-life outcomes in the FIRE AND ICE trial. Eur Heart J. 2016; Epub ahead of print.

\section{Kommentar}

Diese erste große Vergleichsstudie zeigt hinsichtlich patientenorientierter Endpunkte wie der Rehospitalisierungsrate oder erneuter Ablationsmaßnahmen Vorteile des Kryoballons gegenüber dem bislang vornehmlich verwendeten Verfahren der Radiofrequenzablation bei selektierten Patienten mit symptomatischem Vorhofflimmern. Die Kryoballon-Therapie ist daher eine valide Alternative für diese Patientengruppe.

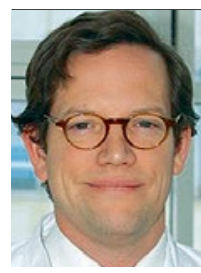

Prof. Dr. Peter W. Radke Klinik für Innere Medizin - Kardiologie Schön Klinik Neustadt

Bei keinem der Erfolgskriterien wie Dauer des Klinikaufenthalts, Sinusrhythmus bei Klinikentlassung oder nach 60 Tagen und auch nicht bei den schweren Nebenwirkungen wie Todesfälle, Rehospitalisierung, Thromboembolie, schwere Blutung, Infektion, Herzrhythmusstörung und Pleuraerguss gab es signifikante Unterschiede (siehe Tabelle 1).

Wie bei solchen Studien üblich und im Protokoll vorgesehen, wechselten einige Patienten ihre zugeteilte Gruppe: $26,7 \%$ in der Frequenz- und $24 \%$ in der Rhythmusgruppe, überwiegend weil das Herzfrequenzziel unter 100/Minute nicht erreicht wurde bzw. weil Nebenwirkungen unter Amiodaron auftraten. Alle Patienten mit Vorhofflimmern von mehr als 48 Stunden oder mit mindestens zwei Perioden wurden antikoaguliert.

Gillinov AM et al. Rate Control versus Rhythm Control for Atrial Fibrillation after Cardiac Surgery. N Engl J Med. 2016;374:1911-1921.

\section{Kommentar}

Postoperatives Vorhofflimmern ist häufig. Es erhöht das Risiko von Komplikationen, die 